\title{
Ovarian effects of a high lactose diet in the female rat
}

\author{
Gentao LIU ${ }^{\mathrm{a}}$, Fangxiong SHI ${ }^{\mathrm{b}}$, Uriel BLAS-MACHADO ${ }^{\mathrm{c}}$, Quynh DUONG $^{\mathrm{a}}$, \\ Vicki L. DAVIS ${ }^{\mathrm{d}}$, Warren G. FOSTER ${ }^{\mathrm{e}}$, Claude L. HUGHES ${ }^{\mathrm{f}}, \mathrm{g} *$ \\ ${ }^{a}$ Cedars-Sinai Burns and Allen Research Institute, Cedars-Sinai Medical Center/University of California \\ Los Angeles School of Medicine, CA, USA \\ b College of Animal Science and Technology, Nanjing Agricultural University, Nanjing 210095, China \\ ${ }^{c}$ College of Veterinary Medicine, Oklahoma State University, Stillwater, OK 74078, USA \\ d Duquesne University, Pittsburgh, PA, USA \\ e Obstetrics \& Gynaecology, McMaster University Medical Center, Hamilton, ON, Canada \\ ${ }^{\mathrm{f}}$ Department of Medical \& Scientific Services, Quintiles, Research Triangle Park, NC, USA \\ g Department of Biology, East Carolina University, Greenville, NC, USA
}

(Received 20 October 2004; accepted 14 January 2005)

\begin{abstract}
Young women with galactosemia experience ovarian failure at a very early age raising concern about the ovarian toxicity of galactose. While galactose may be present in the diet as a monosaccharide, it is predominantly derived from cleavage of the disaccharide lactose within the intestine. Our previous studies in animals have shown that high galactose diets inhibit ovarian follicular development and long-term exposure to high lactose diets retards growth of rats. The objective of the present study was to determine whether galactose exposure in the form of dietary lactose mimics the effects found previously with diets rich in galactose. Sixty female Long-Evans rats (25-day-old) were randomly assigned to two groups and fed a control diet $(41.9 \%$ glucose in AIN93G [Ämerican Institute of Nutrition], CON) before lactose treatment. Unilateral ovariectomy (uOVX) was performed on half of the rats in each group to determine baseline ovarian follicle numbers. The study diet was a high lactose diet (HLD) containing 41.9\% lactose in AIN93G. Study diet exposure started 1 month after uOVX (3 months old) and continued for 7 months in the treatment group. The control group remained on the $41.9 \%$ glucose diet throughout. Vaginal cytology, ovarian morphometric analyses, and serum concentrations of estradiol and progesterone were examined. Long-term exposure to the HLD decreased the body weights of animals and progesterone concentrations in the serum but produced no harmful effects on ovarian morphology or function. Beginning at 5 months of age (two months of lactose treatment) increasing numbers of females began to cycle irregularly but there was no difference between the glucose and lactose diet groups. These negative findings imply that administration of galactose in the form of lactose seems to be much less toxic than when galactose is fed to animals. From a human health perspective, these results are somewhat reassuring, since in general, women eat lactose-containing foods rather than foods that contain large amounts of free galactose.
\end{abstract}

lactose / galactose / ovary / toxicology / rat

* Corresponding author: Claude.hughes@quintiles.com 


\section{INTRODUCTION}

Women in Western countries are urged to consume dairy products to enhance their calcium intake, with the anticipated benefit of reduction in risk of osteoporosis [1]. Since the disaccharide lactose, consisting of one glucose molecule and one galactose molecule is the primary carbohydrate in milk, those who consume large quantities of dairy products in an effort to increase calcium intake for osteoporosis prevention ingest large quantities of lactose. The toxic effect of galactose or its metabolites within the ovary became apparent from the observations of extremely early ovarian failure (before age 30 years) in women with galactosemia (genetic absence of the enzyme galactose-1-phosphate uridyl transferase) [2]. Animal studies have suggested that diets rich in galactose produce insults that are at least somewhat comparable to those seen in galactosemics [2-4]. Furthermore, the importance of galactose metabolism for healthy ovarian function is also suggested by the relative abundance of galactose-metabolizing enzymes in ovarian tissue [5]. Subsequently two specific health concerns for all women have been raised: (1) a concern that dietary galactose might increase the risk of ovarian cancer, and (2) the prospect that dietary galactose might accelerate the age of onset of ovarian failure in women in the general population. In both cases, population-based and experimental studies have begun to clarify the extent of any relationship between dietary intake of galactose in the form of lactose and ovarian disease.

Subsequent to an earlier report suggesting a possible link between ovarian cancer and lactose consumption [6], a number of studies designed to specifically test the relationship between lactose from milk products and ovarian cancer have been published [7-15]. Among these, several studies [9-12] have generally not confirmed original suspicions that galactose consumption and/or slow metabolism (i.e. slow galactose transferase activity) are risk factors for ovar- ian cancer. Moreover, a large populationbased case-control study conducted in Los Angeles County, designed to test the hypothesis that galactose consumption, galactose transferase activity, and/or galactose transferase genotype were associated with ovarian cancer risk, failed to find any association [13-15].

Epidemiological data have suggested that an earlier age of menopause can be associated with galactose intake, but perhaps only in those women who have partial deficiencies of the transferase enzyme (approximately $10 \%$ of the population) $[17,18]$. In our previous study of perimenopausal women, increased levels of follicle-stimulating hormone (FSH, a marker of ovarian senescence) were associated with galactose consumption irrespective of transferase activity [8]. The implication is that women ingesting large amounts of galactose may unknowingly foreshorten the functional lifespan of their ovaries. Animal studies showing toxic effects of dietary galactose have used diets rich in galactose [2-4] which differs from the human situation where galactose exposure results from dietary lactose. Thus, discrepancies in the literature regarding galactose and ovarian failure may be attributable to the different forms of the dietary sugar (monosaccharide versus disaccharide) used in animal experiments. Therefore the objective of this study was to address the effects of a high lactose diet on ovarian function in an animal model. Specifically female rats were exposed to diets rich in lactose or glucose.

\section{MATERIALS AND METHODS}

\subsection{Animals and diets}

Intact, immature Long-Evans female rats (25-days-old) were purchased from Harlan Sprague Dawley and maintained in airconditioned quarters with food and water available ad libitum. The lighting schedule 
was $12 \mathrm{~L}: 12 \mathrm{D}$ with lights on 05:00 $\mathrm{h}$ through 17:00 $\mathrm{h}$. The animals were pairhoused in the Cedars-Sinai Vivarium, which is accredited by the International Association for Laboratory Animal Care. All of control and treatment diets were prepared by Harlan Teklad, Madison, WI. All elements of the diet remained constant throughout groups, with the exceptions glucose and lactose content. All animal procedures were conducted in accordance with the Institutional Animal Care \& Use Committee (IACUC) approved protocol.

\subsection{Experimental designs}

To achieve relevant levels of galactose, and to present it in the form of human dietary exposure (lactose), a semisynthetic diet that mimics the AIN (American Institute of Nutrition; now known as the America Society for Nutritional Sciences) 93G diet was used. Sugar intake was modified from the standard $238.6 \mathrm{~g} \cdot 1800 \mathrm{cal}^{-1}$ of sucrose. Given that the energy content of glucose and galactose are identical, glucose was used as the control. In the control diet, rats were given $41.9 \mathrm{~g}$ glucose $\cdot 100 \mathrm{~g}^{-1}$ of diet (CON) and no added lactose. In the treatment group, rats were given $41.9 \mathrm{~g}$ lactose $100 \mathrm{~g}^{-1}$ of diet (HLD) and no added glucose. Sixty intact, immature rats (25-days-old) were fed CON diet upon arrival before lactose treatment. Rats were randomized into two groups based on their body weight. One month later, unilateral ovariectomy (uOVX) was performed on half of the animals in each treatment group. Exposure to the lactose diet was commenced one month after unilateral ovariectomy. Necropsy was performed seven months after the start of treatments. Animals were monitored daily for signs of adverse effects of the diet. Weekly body weights were recorded and at the conclusion of the study animals were euthanized by carbon dioxide inhalation, and blood was withdrawn from the heart. Uteri and ovaries were collected for analysis.

\subsection{Vaginal cytology}

Vaginal cytology was assessed five days per week at baseline and throughout the experiment as a measure of ovarian function and to determine reproductive senescence. The vaginal smears were stained with $1 \%$ toluidine blue and read according to established procedures [16]. Females that exhibited at least 3 consecutive 4 or 5 day cycles were considered to be regularly cyclic, whereas those that displayed prolonged cycles (6-12 days in duration) were classified as irregularly cyclic. Rats that had shown persistent vaginal cornification for at least 15 consecutive days were classified as being in persistent estrus (PE).

\subsection{Hormone measurement}

Serum concentrations of estradiol and progesterone were assayed using a Coat-ACount commercial kit from Diagnostic Products Corp. (Los Angeles, CA). The intraassay coefficients of variation determined in this laboratory for the estradiol and progesterone measurements were $9.2 \%$ and $11.3 \%$, respectively. The inter-assay coefficients of variation were less than $10 \%$. All analyses were performed within 3 months of sample collection.

\subsection{Ovarian morphometric analysis}

Ovaries were fixed in $4 \%$ paraformaldehyde solution, and subsequently transferred to $70 \%$ ethanol after $24 \mathrm{~h}$. The tissue was prepared for light microscopic analysis using routine techniques. Ovaries were serially sectioned through the thickest part of the parenchyma at $10 \mu \mathrm{m}$, mounted 10 sections on one slide, and stained with hematoxylin and eosin.

Criterion for follicle identification are: (i) Primordial follicles: follicles that have an oocyte with no surrounding granulosa cells and extend to those that have a complete single layer of flattened granulosa cells surrounding the oocyte. (ii) Growing follicles: (a) The oocyte has started to grow and 
Table I. Body weights and weights of the ovary and uterus in Long-Evans rats after long-term exposure to high lactose diets.

\begin{tabular}{lcccc}
\hline Weights & $\begin{array}{c}\text { Control* } \\
\text { (intact) }\end{array}$ & $\begin{array}{c}\text { Control } \\
(\mathrm{uOVX})\end{array}$ & $\begin{array}{c}\text { High lactose } \\
\text { (intact) }\end{array}$ & $\begin{array}{c}\text { High lactose } \\
\text { (uOVX) }\end{array}$ \\
\hline Body (g) & $360.9 \pm 14.8$ & $354.70 \pm 11.7$ & $321.30 \pm 9.9^{\mathrm{a}, \mathrm{b}}$ & $311.0 \pm 6.5^{\mathrm{a}, \mathrm{b}}$ \\
One ovary (mg) & $52.5 \pm 5.5$ & $76.73 \pm 6.5$ & $65.1 \pm 5.7$ & $84.1 \pm 5.9$ \\
Uterus (mg) & $649.9 \pm 47.7$ & $566.00 \pm 27.3$ & $750.7 \pm 53.5$ & $654.93 \pm 49.5$ \\
\hline
\end{tabular}

* Each group consisted of fifteen animals.

a $P<0.05$ compared to control (intact).

b $P<0.05$ compared to control (uOVX).

the surrounding granulosa cell-layer has become columnar and extend to those that have formed multiple layers of granulosa and thecal cells. (b) There is no evidence of antrum formation. (iii) Antral follicles: (a) Large oocyte size. (b) Multiple cell layers surrounding the oocyte. (c) Formation of the (fluidfilled) antrum. (iv) Atretic follicles: (a) Cystic follicular degeneration. (b) Oocyte liquefies and zona pellucida becomes thickened and folded. (c) Degeneration and loss of granulosa cells through apoptosis and swelling (oncosis). (d) Invasion of foamy histiocytes into follicular antrum. (e) Wall collapsed. (f) Vascular invasion of follicular antrum. (g) Thecal cell hypertrophy followed by luteinization (cells become pigmented and foamy). (h) Increased histiocytes and fibroblasts. (v) Corpora Hemorrhagica (New CL's). (vi) Corpora Lutea (Old CL's) [17]. The total number of follicles in each group was counted microscopically at $\times 200$ by a blinded observer for each ovary; these means were pooled to obtain group means.

\subsection{Statistical methods}

Statistical analysis of the estrous cycle patterns was performed using the $\chi^{2}$ test. Comparisons of body weight, follicle number and hormone levels were revealed by Two Way ANOVA analysis followed by Tukey Test for multiple comparisons when appropriate. A level of $P<0.05$ was considered statistically significant. The follicle decrease between baseline and the end of the exper- iment was analyzed by paired $t$-test. Statistical calculations were performed using Sigmastat for Windows, version 2.03 (Jandel Scientific Corporation Sigma Stat 2.03). Data are presented as the mean \pm SEM.

\section{RESULTS}

\subsection{Effects of dietary lactose on vaginal cytology at different ages}

Before 5 months of age, all rats had regular estrous cycles. Afterwards, the incidence of irregular cyclicity appeared and increased. More animals in the HLD group had regular estrous cycles compared to the control group. However, no significant differences among the treatment groups could be demonstrated (data not shown). Also, there were no effects of uOVX on the estrous cycle of rats in this experiment (data not shown).

\subsection{Effects of dietary lactose on the body weight, weights of ovary and uterus}

In intact females the body weight from the lactose treated group was significantly lower than that of the control group. Fresh ovarian weight was greater in the HLD group, but no significant difference was found between treatment groups. The ovarian weights from uOVX control and HLD rats were greater than those of the intact rats (Tab. I). There was no difference in uterus 
Table II. Serum concentrations of progesterone and estradiol in Long-Evans rats after long-term exposure to high lactose diets.

\begin{tabular}{lccc}
\hline Group & $N$ & $\begin{array}{c}\text { Progesterone } \\
\left(\mathrm{ng} \cdot \mathrm{mL}^{-1}\right)\end{array}$ & $\begin{array}{c}\text { Estradiol } \\
\left(\mathrm{pg} \cdot \mathrm{mL}^{-1}\right)\end{array}$ \\
\hline $\begin{array}{l}\text { Control } \\
\text { (intact) }\end{array}$ & 15 & $12.2 \pm 2.4$ & $63.5 \pm 3.7$ \\
$\begin{array}{l}\text { Lactose } \\
\text { (intact) }\end{array}$ & 15 & $8.0 \pm 1.0^{\mathrm{A}}$ & $60.1 \pm 3.8$ \\
$\begin{array}{l}\text { Control } \\
\text { (uOVX) }\end{array}$ & 15 & $10.7 \pm 2.1$ & $68.7 \pm 2.3$ \\
$\begin{array}{l}\text { Lactose } \\
\text { (uOVX) }\end{array}$ & 15 & $7.7 \pm 1.6^{\mathrm{B}}$ & $68.1 \pm 5.7$ \\
\hline
\end{tabular}

A Denotes $P<0.01$ compared with the group of intact rats fed control diet.

B Denotes $P<0.01$ compared with the group of OVX rats fed control diet.

weight among any of the groups. Histopathological assessment of the ovaries and uterus failed to reveal any hyperplastic or neoplastic lesions in any of the rats.

\subsection{Effects of dietary lactose on serum concentrations of progesterone and estradiol}

The difference in the mean progesterone concentrations among the different diets was greater than would be expected by chance after allowing for effects of differences in OVX. The progesterone levels were significantly decreased $(P<0.01)$ after lactose treatment whether the rats were intact or OVX. However, there were no significant differences in serum concentrations of estradiol between treatment groups (Tab. II).

\subsection{Effects of dietary lactose on ovarian histological features}

The ovaries collected from rats at the time of unilateral ovariectomy at 2 months of age were analyzed. Ovarian follicles were counted to establish a baseline against which to assess the effects of a HLD on follicle reserve. Baseline follicle counts were equiv- alent in the two groups before lactose treatment (Tab. III, Part I).

Following seven months of treatment, intact animals had more primordial follicles (within a single ovary) than uOVX rats had in the remaining ovary $(P<0.01)$ (Tab. III, Parts II and III). There were no significant differences in the number of primordial, growing, and antral follicles among treatment groups within the respective category. Follicle numbers of growing follicles and antral follicles in all rats decreased significantly at the end of this study compared to those of baseline $(P<0.05)$. However, there were no differences between different diets.

\section{DISCUSSION}

Previously, we reported the metabolic effects of dietary lactose in adult female rats [18], the objective of the present study was to determine the ovarian effects associated with prolonged exposure to galactose when it is provided as a high lactose diet (HLD) which is more representative of human exposure than experimental diets used in prior studies that contained galactose per se. Results of our study show that female rats fed a HLD for seven months had decreased body weights and serum progesterone concentrations, however, these animals did not develop ovarian dysfunction. Furthermore, cataracts are a known adverse effect of high galactose exposure [21, 22], but no cataracts were observed in the present study (data not shown). Together, these findings imply that administration of galactose in the form of a HLD is less toxic than when galactose per se is fed to animals.

Whether these results can be extrapolated to women will require further study. The ovary has relatively abundant levels of three of the major enzymes involved in metabolism of galactose (galactokinase, galactose-1-phosphate uridyltransferase (GALT) and UDP-galactose-4-epimerase) [6]. This fact is thought to be causally related to the observation that women who have absent or 
Table III. Follicle numbers before and after 7 months of lactose treatment ${ }^{1}$.

\begin{tabular}{lcccccc}
\hline $\begin{array}{l}\text { Part } \\
\text { of table }\end{array}$ & Group & $\begin{array}{c}\text { Section area } \\
\left(\mathrm{mm}^{2}\right)\end{array}$ & Primordial follicle & $\begin{array}{c}\text { Growing } \\
\text { follicle }\end{array}$ & $\begin{array}{c}\text { Antral } \\
\text { follicle }\end{array}$ & $\begin{array}{c}\text { Atretic } \\
\text { follicle }\end{array}$ \\
\hline I & $\begin{array}{c}\text { Baseline of } \\
\text { control group } \\
\text { Baseline of } \\
\text { lactose group }\end{array}$ & $9.4 \pm 1.20$ & $16.6 \pm 1.3^{\mathrm{a}}$ & $7.9 \pm 1.1^{\mathrm{b}}$ & $7.1 \pm 0.9^{\mathrm{b}}$ & $33.6 \pm 4.2$ \\
& $9.7 \pm 1.0$ & $16.7 \pm 2.8^{\mathrm{a}}$ & $7.5 \pm 0.8^{\mathrm{b}}$ & $7.7 \pm 1.0^{\mathrm{b}}$ & $37.6 \pm 2.2$ \\
$\mathrm{II}$ & $\begin{array}{l}\text { Control } \\
\text { (intact) }\end{array}$ & $9.1 \pm 1.2$ & $14.8 \pm 2.07$ & $5.3 \pm 0.7$ & $2.9 \pm 0.6$ & $42.4 \pm 5.5$ \\
& $\begin{array}{l}\text { Lactose } \\
\text { (intact) }\end{array}$ & $9.5 \pm 1.4$ & $14.0 \pm 1.7$ & $6.5 \pm 3.0$ & $1.8 \pm 0.8$ & $24.4 \pm 11.6$ \\
\hline & $\begin{array}{l}\text { Control } \\
(\text { uOVX) }\end{array}$ & $9.9 \pm 1.9$ & $6.1 \pm 1.4^{\mathrm{c}}$ & $3.7 \pm 0.7$ & $2.1 \pm 0.7$ & $29.1 \pm 4.9$ \\
III & $\begin{array}{l}\text { Lactose } \\
(\text { uOVX) }\end{array}$ & $11.4 \pm 1.3$ & $6.9 \pm 0.6^{\mathrm{c}}$ & $4.8 \pm 0.9$ & $1.6 \pm 0.8$ & $31.6 \pm 5.3$ \\
& & & & & \\
\hline
\end{tabular}

${ }^{1}$ The follicle number was counted on the largest section of ovary. Data represent the mean \pm SEM of 15 animals per treatment group.

${ }^{2}$ The baseline follicle numbers for control and lactose groups were counted on the ovarian tissue obtained by OVX before lactose treatment.

a Denotes $P<0.05$ compared with two different groups in Part III.

b Denotes $P<0.05$ compared with four different groups in Parts II and III.

${ }^{\mathrm{c}}$ Denotes $P<0.01$ compared with two different groups in Part II.

low GALT activity have a propensity to develop premature ovarian failure and premature menopause [2, 17]. However, since human diets contain foods high in lactose versus free galactose, our data are suggestive that women would not have the adverse effects observed in rodents that consume a high galactose diet.

We used traditional vaginal cytology to assess the effects of lactose on the onset of reproductive senescence. Female rats usually display regular vaginal cycles beginning at around 45 days of age; however, aging female rats exhibit a progressive decrease in their abilities to maintain regular estrous cycles [19] and fertile gestations. Previous studies demonstrate that beginning at middle age, many female rats display prolonged, irregular cycles instead of regular cyclicity $[20,21]$. The present study demonstrated that the incidence of both regular estrous cycles and follicle numbers decreased markedly between 5 and 10 months of age in the Long-Evans rat exposed to either a glucose- rich control diet or a lactose-rich study diet. There was no difference among the treatment groups although the HLD group appeared to have a lower incidence of irregular estrous cycles. The progression of spontaneous ovarian failure in these rats with diminished ovarian reserve due to the initial unilateral ovariectomy has not been previously characterized, although the time course of spontaneous follicular depletion and cessation of cyclicity in intact rats of this strain are well studied. In a previous study [22], only $51 \%$ of the virgin LongEvans rats, fed regular chow, maintained regular estrous cycles, and $42 \%$ of them had fertile gestations after mating at 8 months of age. By 10 months of age, the incidence of regular cyclicity dropped to $38 \%$. Results from the present study are therefore consistent with prior studies in Long-Evans rats. Therefore, we propose that the decline in regular frequency of estrous cycles in the present study is the naturally-occurring phenomenon associated with aging in LongEvans rats. 
Findings from our previous study in Japanese quail [23] and from three rodent studies reported by other groups [2-4] demonstrate that galactose exerts toxic effects on the ovary. Moreover, female rat fetuses exposed to high levels of galactose $(50 \%$ in maternal diet) during gestation had a significantly decreased number of oocytes [2]. Swartz and Mattison observed a number of effects on the ovaries of adult female mice fed a $40 \%$ galactose diet for two weeks [3]. These changes included inhibition of oocyte maturation shown by a decreased number of corpora lutea, a decreased response to superovulatory stimuli, an increase in interstitial tissue and lipofuscin staining and finally a failure to respond to exogenous gonadotropins. Similar effects were also reported in prepubertal rats [4]. In our shortterm study, we found that Long-Evans rats fed $20 \%$ galactose for 19 days developed cataracts and had a significant decrease in the number of growing antral follicles (unpublished data). However in this study, treatment with a HLD equivalent to $20 \%$ galactose for seven months did not induce follicle depletion compared to the control group, which supports the premise that the toxicity of lactose is much less than galactose [24]. When galactose is absorbed along with glucose, the serum galactose concentrations are considerably lower than when the same amount of galactose is consumed without glucose and that glucose increases GALT activity in the liver [25]. Galactose and glucose share an intestinal transport carrier and this transport carrier has a greater affinity for glucose than galactose [26] which may explain why galactose absorption is inhibited by glucose but glucose absorption is unaffected by galactose [27]. In addition to the decrease in intestinal absorption of galactose by glucose, it is thought that the insulin response to glucose increases the rate of clearance and metabolism of galactose [24]. Since digestion of lactose results in the simultaneous production of glucose and galactose, these mechanistic findings suggest that consumption of lactose would produce less toxicity than diets containing only galactose. Our results reported herein in the rat model are consistent with that viewpoint, in that a high lactose diet did not induce ovarian failure as was observed with high galactose diets [3-5].

In summary, our results show that longterm exposure to a high lactose diet decreased the body weights of animals, but produced no harmful effects on ovarian morphology or function. Beginning at 5 months of age (two months of lactose treatment) increasing numbers of females began to cycle irregularly but there was no difference between the glucose and lactose diet groups. These negative findings imply that administration of galactose in the form of lactose seems to be much less toxic than when galactose per se is fed to animals. From a human health perspective, these reassuring results suggest that ovarian function should not be impaired in women, since their primary source of galactose would come from the metabolism of lactose in foods, such as dairy products.

\section{ACKNOWLEDGMENTS}

This study was supported by NIH grant (R03HD350830) - Ovarian Toxicity of Galactose (Claude Hughes).

\section{REFERENCES}

[1] Soroko S, Holbrook TL, Edelstein S, BarrettConnor E. Lifetime milk consumption and bone mineral density in older women. Am J Public Health 1994, 84: 1319-1322.

[2] Chen YT, Mattison DR, Feigenbaum L, Fukui $\mathrm{H}$, Schulman JD. Reduction in oocyte number following prenatal exposure to a diet high in galactose. Science 1981, 214: 1145-1147.

[3] Swartz WJ, Mattison DR. Galactose inhibition of ovulation in mice. Fertil Steril 1988, 49: 522-526.

[4] Meyer WR, Doyle MB, Grifo JA, Lipetz KJ, Oates PJ, DeCherney AH, Diamond MP. Aldose reductase inhibition prevents galactoseinduced ovarian dysfunction in the SpragueDawley rat. Am J Obstet Gynecol 1992, 167: 1837-1843. 
[5] Xu YK, Ng WG, Kaufman FR, Lobo RA, Donnell GN. Galactose metabolism in human ovarian tissue. Pediatr Res 1989, 25: 151-155.

[6] Cramer DW, Harlow BL, Willett WC, Welch WR, Bell DA, Scully RE, Ng WG, Knapp RC. Galactose consumption and metabolism in relation to the risk of ovarian cancer. Lancet 1989, 2: 66-71.

[7] Cooper GS, Hulka BS, Baird DD, Savitz DA, Hughes CL Jr, Weinberg CR, Coleman RA, Shields JM. Galactose consumption, metabolism, and follicle-stimulating hormone concentrations in women of late reproductive age. Fertil Steril 1994, 62: 1168-1175.

[8] Robb-Nicholson C. I read recently that there may be a connection between ovarian cancer and the consumption of dairy products, particularly cottage cheese and yogurt. Has this connection been proven? Harv Womens Health Watch 1998, 6: 8.

[9] Engle A, Muscat JE, Harris RE. Nutritional risk factors and ovarian cancer. Nutr Cancer 1991, 15: 239-247.

[10] Mettlin CJ. Invited commentary: progress in the nutritional epidemiology of ovary cancer. Am J Epidemiol 1991, 134: 457-459; discussion 460-451.

[11] Webb PM, Bain CJ, Purdie DM, Harvey PW, Green A. Milk consumption, galactose metabolism and ovarian cancer (Australia). Cancer Causes Control 1998, 9: 637-644.

[12] Risch HA, Jain M, Marrett LD, Howe GR. Dietary lactose intake, lactose intolerance, and the risk of epithelial ovarian cancer in southern Ontario (Canada). Cancer Causes Control 1994, 5: 540-548.

[13] Goodman MT, Wu AH, Tung KH, McDuffie $\mathrm{K}$, Cramer DW, Wilkens LR, Terada K, Reichardt JK, Ng WG. Association of galactose-1-phosphate uridyltransferase activity and N314D genotype with the risk of ovarian cancer. Am J Epidemiol 2002, 156: 693-701.

[14] Fung WL, Risch H, McLaughlin J, Rosen B, Cole D, Vesprini D, Narod SA. The N314D polymorphism of galactose-1-phosphate uridyl transferase does not modify the risk of ovarian cancer. Cancer Epidemiol Biomarkers Prev 2003, 12: 678-680.

[15] Cozen W, Peters R, Reichardt JK, Ng W, Felix JC, Wan P, Pike MC. Galactose-1-phosphate uridyl transferase (GALT) genotype and phenotype, galactose consumption, and the risk of borderline and invasive ovarian cancer (United States). Cancer Causes Control 2002, 13: $113-120$
[16] Cooper RL, Goldman JM, Vandenbergh JG. Monitoring of the estrous cycle in the laboratory rodent by vaginal lavage. In: Heindel JJ, Chapin RE (Eds), Female Reproductive toxicity, Academic Press, Inc, New York, 1993, p 45-56.

[17] Plowchalk DR, Smith B, Mattiso DR. Assessment of toxicity to the ovary using follicle quantitation and morphometrics. In: Heindel JJ, Chapin RE (Eds), Methods in ToxicologyFemale Reproductive Toxicology, Academic Press, Inc, 1993, p 57-68.

[18] Liu G, Hughes CL, Mathur R, Foster WG, Davis VL, Magoffin DA. Metabolic effects of dietary lactose in adult female rats. Reprod Nutr Dev 2003, 43: 567-576.

[19] Lu JK, LaPolt PS, Nass TE, Matt DW, Judd HL. Relation of circulating estradiol and progesterone to gonadotropin secretion and estrous cyclicity in aging female rats. Endocrinology 1985, 116: 1953-1959.

[20] Anzalone CR, Lu JK, LaPolt PS. Influences of age and reproductive status on ovarian ovulatory responsiveness to gonadotropin stimulation. Proc Soc Exp Biol Med 1998, 217: 455460.

[21] LaPolt PS, Matt DW, Lu JK. Progesterone implants delay age-related declines in regular estrous cyclicity and the ovarian follicular reserve in Long-Evans rats. Biol Reprod 1998, 59: 197-201.

[22] Matt DW, Server PL, Lu JK. Relation of parity and estrous cyclicity to the biology of pregnancy in aging female rats. Biol Reprod 1987, 37: 421-430.

[23] Liu GG, Huang H, Wang RL, Hughes CL. Taurine prevents ovarian toxicity of galactose in Japanese Quail. Jiangsu J Agric Sci 2001, 17: 44-48.

[24] Williams CA, Phillips T, Macdonald I. The influence of glucose on serum galactose levels in man. Metabolism 1983, 32: 250-256.

[25] Rogers S, Segal S. Changing activities of galactose metabolizing enzymes during perfusing of suckling rat liver. Am J. Physiol 1981, 240: 333-339.

[26] Crane RK. Digestion and absorption: water soluble organics. International Review of Physiology, 12th ed, Crane RK (Ed), University Park Press, 1977.

[27] Holdsworth CD, Dawson AM. The absorption of monosaccharides in man. Clin Sci 1964, 27: 371-379. 